\title{
The emergence of virulence via the collective action of bacterial effectors
}

David Guttman ( $\nabla$ david.guttman@utoronto.ca )

University of Toronto https://orcid.org/0000-0001-8479-3869

\section{Tatiana Ruiz-Bedoya1}

University of Toronto

\section{Pauline Wang}

University of Toronto

Darrell Desveaux

University of Toronto

\section{Article}

Keywords:

Posted Date: January 19th, 2022

DOI: https://doi.org/10.21203/rs.3.rs-1159977/v1

License: (c) (1) This work is licensed under a Creative Commons Attribution 4.0 International License.

Read Full License 


\section{Affiliations:}

$6{ }^{1}$ Department of Cell \& Systems Biology, University of Toronto, 25 Willcocks Street, Toronto, 7 ON M5S 3B2, CAN

$8 \quad{ }^{2}$ Centre for the Analysis of Genome Evolution \& Function, University of Toronto, 25, Willcocks

9 Street, Toronto, ON M5S 3B2, CAN

10

11

$12 *$ Correspondence to: darrell.desveaux@utoronto.ca (D.D.); david.guttman@utoronto.ca (D.S.G)

$13 \uparrow$ These authors contributed equally and are listed in alphabetical order. 


\section{Summary}

Eukaryotes continuously interact with diverse microbial populations and communities, forming relationships spanning a continuum from beneficial to pathogenic. Pathogenic effectors are central to these associations since they directly target host cellular components to promote microbial fitness ${ }^{1,2}$. Although effectors are necessary for virulence and well-studied from the context of single pathogenic strains ${ }^{3,4}$, the extent to which they can modulate population or community dynamics is largely unknown. Here we show that type III effectors of the bacterium Pseudomonas syringae act as public goods to promote the emergence of collective virulence in the plant Arabidopsis thaliana. We disaggregated the effector suite of a $P$. syringae strain highly virulent on $A$. thaliana into a coisogenic population with each strain carrying only one effector, (i.e., a metaclone), and showed that while each coisogenic strain in the metaclone is individually unfit, the metaclone cooperates mutualistically to fully restore virulence. We also show that this state of collective virulence emerged irrespective of the microbe's genetic background, being capable of transforming the rhizosphere-inhabiting, beneficial bacterium Pseudomonas fluorescens ${ }^{5}$ into a foliar pathogen. These results expand our knowledge of the mechanisms available to polyclonal populations and polymicrobial communities to exploit hosts. They also raise potential evolutionary routes for the emergence of new host-specific pathogens and expand the ecological scale at which disease may be attributed. 


\section{Main Text}

Disease is typically thought to be driven by the colonization, growth, and/or dissemination of a single pathogenic organism; a view originating from the work of Koch and Henle that established postulates for ascribing causative relationships between microbes and diseases ${ }^{6,7}$. Despite the foundational nature of Koch's postulates, they are generally considered incomplete unless adapted to include the complex and interdependent roles of the host, the environment, and polyclonal (i.e., intra-specific) or polymicrobial (i.e., inter-specific) diversity, i.e., hostassociated microbiota. We now know that while disease may be driven by a single pathogenic clone, this clone is almost always embedded in a diverse microbial community that includes many interacting species as well as other conspecific lineages ${ }^{8-10}$. Infections encompassing these diverse communities have the potential for collective or social behaviors not available to single clone infections. These social interactions can result in non-additive, or emergent outcomes that dramatically change the fitness of individual members of the community. For example, a strain lacking required virulence factors will have low fitness in isolation but can achieve much higher fitness in the presence of an adapted strain by taking advantage of the permissive environment created by the latter ${ }^{11,12}$.

Many bacterial pathogens require a T3SS and a suite of associated type III secreted effectors (hereafter effectors) to interact with their eukaryotic hosts. Extracellular and membrane-bound intracellular pathogens inject suites of effectors directly into host cells to manipulate cellular processes and promote immunosuppression and nutrient acquisition ${ }^{1,2}$. In plants, effector activities are closely monitored by the host immune system, which can mount an effector- 
triggered immune (ETI) response if the host encodes immune receptors that recognize the presence or activity of specific effectors ${ }^{13}$. Consequently, the specific suite of effectors carried by an individual strain largely determines whether its interaction will result in disease or immunity on a particular host. Bacterial effectors injected into host cells act intracellularly, but the immune-modulatory and/or nutritional benefits can be accrued by all microbes in the vicinity of the breached host cell. Given this, effectors could reasonably be expected to act as 'public goods', which are products (i.e., molecules or functions) that are costly to produce and benefit both the producer as well as other members in the population or community ${ }^{14-16}$. Strains that produce a public good are sometimes called actors, while those that benefit from a public good but do not produce it are called cheaters ${ }^{16}$. Since public goods are, by definition, costly to produce, a cheater that does not produce the public good will have a competitive advantage over the actor. Despite the competitive advantage of cheaters, multiple evolutionary models of virulence describe how cooperation and coexistence can be maintained in a population ${ }^{11,17}$.

The T3SS provides an outstanding opportunity to study social interactions among bacteria, and multiple studies have shown that a T3SS deficient cheater strain gains a strong fitness benefit when grown with a T3SS wildtype actor strain $^{11,12}$. One example is the common bacterial plant pathogen Pseudomonas syringae, which requires a functional T3SS and appropriate suite of effectors to cause disease. P. syringae is a highly diverse species complex that causes disease on a wide range of plant species, including many of agricultural importance ${ }^{18-21}$. The P. syringae complex encodes over 5000 unique effector proteins in its pangenome distributed among 70 distinct effector families, with most strains carrying a suite of 12 to 40 effectors ${ }^{22}$. The $P$. 
syringae T3SS and its effectors are required for virulence ${ }^{23,24}$, but interestingly, a T3SS knockout mutant strain can cheat when in a mixed infection with a T3SS wildtype strain and grow to near wildtype levels ${ }^{25}$. Similar T3SS-associated cheating behavior has also been seen with human pathogens ${ }^{12}$. While these studies clearly demonstrate that a T3SS mutant cheater benefits from the presence of the T3SS wildtype actor, they do not demonstrate that the success of the population requires the collective action of a suite of effectors acting as public goods. Nor do these studies show that this collective action can give rise to virulence as an emergent property in a population where no individual strain has this capability.

Here we show that effectors act as public goods even when effector suites are completely disaggregated into a coisogenic population that we call a metaclone. Strains in a metaclone share the same genetic background and are only differentiated by a single expressed effector (Fig. 1a). Metaclones provide a means to perform fine-scale dissection of naturally occurring and synthetic effector suites and quantify fitness contributions of individual effectors, combinations of effectors, and effector-effectors interactions. We used metaclones to show that a population of 35 coisogenic strains each carrying one effector (i.e., a disaggregated effector suite) was equally virulent as the parental strain carrying the same 35 effectors. In this case, however, metaclone virulence requires collective behavior and mutualistic interactions among strains. We also show that the public good benefits of effectors can be ported to a highly divergent con-specific strain indicating that they function independently of the genetic background. Finally, we show that this portability is even effective when the effectors are delivered from a different species. We converted the beneficial bacterial species, Pseudomonas fluorescens, into a pathogen by creating 
101 a $P$. fluorescens metaclone carrying $P$. syringae effectors, also demonstrating that effectors are

102 sufficient for virulence. Overall, this work demonstrates the evolutionary and ecological

103 potential conferred by the collective action of pathogen effectors.

\section{Disaggregated effector metaclones}

We developed an experimental system that allows us to evaluate the outcome of effectormediated interactions among con-specific clones via the decentralized delivery of effectors in a synthetic microbial population we call metaclones. We generated the metaclones for this study by chemically synthesizing all 36 well-expressed effector genes annotated in the genome of $P$. syringae pv. tomato DC3000 (PtoDC3000) ${ }^{26}$; a tomato pathogen that is highly virulent on transformed each effector construct into a PtoDC3000 strain from which all 36 effectors have been deleted, called PtoDC3000D36E (hereafter D36E) ${ }^{27}$. While PtoDC3000 can achieve very

114 high levels of growth on A. thaliana, D36E is non-virulent and typically grows three or more 115 orders of magnitude less than PtoDC3000 in standard in planta growth assays. The

116 transformation of individual effectors into D36E generated a library of clones expressing

117 individual effectors that can then be collectively assembled into metaclones (Fig. 1a).

\section{The emergence of collective virulence}

We first recapitulated previous observations that no individual PtoDC3000 effector is sufficient

121 for restoring wildtype levels of virulence when expressed in the effectorless D36E background 
3,4,24,27-30. To do this, we performed A. thaliana virulence assays via spray inoculations for each of the 36 PtoDC3000 effectors individually cloned into D36E and compared their growth to that observed with the D36E and parental PtoDC3000 empty vector (EV) strains. While there was minor variation in the growth of the 36 effector clones, none grew to a level statistically different than D36E (Extended Data Fig. 1). We also showed that in vitro growth does not vary significantly among the effector clones (Extended Data Fig. 2).

To test the public nature and collective activity of effectors we disaggregated the PtoDC3000 effector arsenal and asked if a $P$. syringae metaclone composed of all well-expressed PtoDC3000 effectors could achieve the same level of virulence as the wildtype PtoDC3000 strain. In other words, can a population of 36 coisogenic clones each carrying one effector reach the same level of virulence as one strain carrying 36 effectors? Given that no individual effector can restore full virulence, the disaggregated effector arsenal of the PtoDC3000 metaclone could only recapitulate PtoDC3000 virulence if part or all of the metaclone acted collectively. We assembled a PtoDC3000 metaclone called D36E::McDC[36], which comprises a population of all 36 clones individually expressing one of each of the well-expressed PtoDC3000 effectors deleted from D36E. We also assembled a metaclone called D36E::McDC[35] lacking the effector AvrE1f since prior work has shown that this effector can trigger ETI in A. thaliana ecotype Col-0 when expressed on a multicopy plasmid ${ }^{26}$ (Fig. 1b). We spray-infected A. thaliana plants with the two metaclones, the parental PtoDC3000 strain and the D36E effectorless strain harboring empty vectors and quantified in-planta bacterial growth five and seven days post infection. The growth of the non-ETI-eliciting metaclone D36E::McDC[35] was indistinguishable from that of the 
144 virulent parental strain PtoDC3000::EV. In contrast, the growth of the ETI-eliciting metaclone D36E::McDC[36] was indistinguishable from the non-virulent D36E::EV (Fig. 1b). The metaclone D36E::McDC[35] also reached high virulence when using a traditional pressureinfiltration infection method, showing that the results are independent of the means of infection (Extended Data Fig. 4). We confirmed that all individual effector clones were present throughout the infection and the overall diversity of metaclones remained unchanged by tracking unique barcode incorporated into the synthesized sequences (Extended Data Fig. 2). These data show that no individual effector clone dominated the population and that the clones (or at least a subset) act collectively to promote population fitness. The difference in growth observed between D36E::McDC[36] and D36E::McDC[35], which differ only in the presence of a clone carrying the ETI-eliciting AvrE1f, illustrates that effectors imposing a fitness detriment can reduce the fitness of the entire population, in other words, act as a public bad ${ }^{31}$.

We have shown above that individual effector clones within the metaclone can only achieve high fitness through collective action; therefore, virulence in this system is an emergent property of the metaclone population. The low fitness of individual effector clones and high fitness of the metaclone also indicates that obligate mutualistic interactions are occurring among at least a subset of effector clones to achieve virulence. We also presume that not all the individual effector clones are contributing to metaclone fitness, and are therefore, effectively acting as cheaters. Nevertheless, to directly show that the metaclone supports the fitness of a true cheater we included the effectorless strain D36E::EV in the metaclone and assessed its fitness after spray inoculation onto A. thaliana (Fig. 2a). D36E::EV showed significantly enhanced growth when 
accompanied by the $\mathrm{D} 36 \mathrm{E}:: \mathrm{McDC}[35]$ metaclone in comparison to the control effector-less population (Fig. 2b), indicating that the collective virulence of the metaclone is indeed susceptible to exploitation by cheats. It should be emphasized that in this cheater assay, no individual actor strain is providing the public goods since we are working with a disaggregated population of individual effectors that individually have low fitness; the actor is the collective action of the metaclone.

\section{Portability of effector arsenals}

We wanted to test if the collective fitness benefits of the metaclone were portable or transferable to another genetic background. This is particularly relevant given the very high level of recombination and horizontal gene transfer observed among effectors in the $P$. syringae species complex ${ }^{18}$. We created a metaclone composed of well-expressed effectors of $P$. syringae pv. maculicola ES4326 (PmaES4326), which were individually transformed into D36E. PmaES4326 is a radish pathogen in phylogroup 5 that is also strongly virulent on $A$. thaliana but highly divergent from the phylogroup 1 strain PtoDC3000 both with respect to its core genome $\left(\mathrm{d}_{\mathrm{N}}=\right.$ 0.034; $\left.\mathrm{d}_{\mathrm{S}}=0.474\right)$ and effector arsenal (Fig. 3a-c). The combined effector suites carried by PmaES4326 and PtoDC3000 include 43 of the 70 distinct $P$. syringae effector families, with 36 effector families present in PtoDC3000, 26 in PmaES4326, and 19 found in both (Jaccard similarity $=0.44)($ Fig. 3c). At the allelic level, PtoDC3000 and PmES4326 carry 36 and 30 alleles with confirmed expression respectively, ${ }^{27}$, with eight alleles shared by both strains $(\text { Jaccard similarity }=0.14)^{22}$ (Fig. 3b). 
Paralleling the PtoDC3000 metaclones, we created two PmaES4326 metaclones in the D36E background: one with the full complement of effectors, including ETI-elicitors, and one without ETI-elicitors. Two alleles harbored naturally in PmaES4326 (AvrE1i and HopO1b) have the potential to trigger ETI in A. thaliana ${ }^{32}$, so we created an ETI-eliciting metaclone, D36E::McES[30], and a non-ETI eliciting metaclone, D36E::McES[28] (Fig. 3c). Spray inoculation of the metaclones and controls onto A. thaliana showed similar results to what was observed in PtoDC3000 metaclones. The non-ETI-eliciting PmaES4326 metaclone D36E::McES[28] significantly restored in-planta growth compared to the effector-less D36E::EV background strain, whereas the ETI-eliciting metaclone D36E::McES[30] did not (Fig. 2d). These data indicate that the collective action of effectors in a metaclone is functionally independent of the genetic background in conspecific pathogens and that effectors are effectively exchangeable at a relatively large evolutionary distance.

An important question arising from our study of the PmaES3426 metaclone is if the apparent interchangeability of effector arsenals can be explained by the collective action of the seven nonETI eliciting effector alleles that are common to both PtoDC3000 and PmaES4326 metaclones. We tested this by creating D36E::McDC\&ES[7], which is a metaclone composed of the seven shared alleles that do not elicit ETI: HopAA1b, HopAD1a, HopD1a, HopD1e, HopQ1a, HopT1a, and HopV1d (Data S1). As expected, D36E::McDC\&ES[7] failed to recover the same population-level virulence observed for D36E::McDC[35] or the parental strain (Extended Data Fig. 5). Consequently, the restoration of virulence in the PmaES4326 metaclone cannot be explained solely by effectors common to both pathogens PtoDC3000 and PmaES4326. 
211 The sufficiency of effectors for virulence

212 Finally, we tested if disaggregated effector arsenals from a pathogenic species are sufficient to 213 convert a beneficial bacterial species into a pathogen. Pseudomonas fluorescens is a common 214 inhabitant of the plant root microbiome (i.e., the rhizosphere) that has been widely studied as a 215 plant growth-promoting species ${ }^{33-35}$. While $P$. syringae and $P$. fluorescens are sister species 216 complexes, the two lineages are phylogenetically very distinct, with PtoDC3000 and $P$.

217 fluorescens Pf0-1 sharing an average nucleotide identity (ANI) of only $80.73 \%$. This is 218 comparable to Escherichia coli K-12 substr. MG1655 and Salmonella enterica subsp. enterica 219 serovar Typhimurium str. LT2 which have an ANI of 81.91\%. To test our hypothesis, we used 220 the $P$. fluorescens strain Pf01EtHAn, which has been engineered to contain a functional $P$. 221 syringae T3SS capable of delivering effectors ${ }^{5}$. We individually transformed all PtoDC3000 and 222 PmaES4326 effectors into Pf01EtHAn and assembled ETI-eliciting PfEtHAn::McDC[36] and PfEtHAn::McES[30], and non-ETI-eliciting PfEtHAn::McDC[35] and PfEtHAn::McES[28] metaclones. We then quantified their growth in planta relative to their corresponding pathogenic parental strains. Remarkably, the non-ETI eliciting PfEtHAn::McDC[35] metaclone grew to a level indistinguishable from PtoDC3000::EV, while the PfEtHAn::McES[28] metaclone grew to

227 a level indistinguishable from PmaES4326::EV (Fig. 4). Consistent with our previous observations, ETI-eliciting metaclones did not grow significantly better than the effector-less 229 background strains D36E::EV and PfEtHAn::EV. These results demonstrate that the virulence 230 contribution of effectors is transferrable to different species, and that these arsenals (along with 231 the appropriate secretion system) are highly portable and sufficient for pathogenicity. 


\section{Discussion}

While the limitations of Koch's postulates, the importance of polymicrobial and polyclonal infections, and complex interactions in the microbiome are now well recognized, most studies of infectious disease etiology still focus on the role of single, virulent strains ${ }^{8-10,36-40}$. Here, we aimed to show that virulence can be achieved through collective population behavior even in the absence of any individually fit strain. Four main principles arise from these results: (1) Virulence can be a collective phenotype; (2) To act as public goods, effectors do not require centralized delivery and consequently, virulence can be an emergent property; (3) Effector arsenals are highly portable and can act largely independently of the genetic background; (4) The position of a species on the interaction continuum that includes beneficial and virulent microbes is quite labile since a beneficial species can be transformed into a pathogen if provided the appropriate effectors and secretion system.

We demonstrate that effectors are public goods by working with a coisogenic population (i.e., metaclone) that is only able to achieve high virulence through collective action. Hence, all, or a subset, of the population are actors that can only achieve high fitness if they act mutualistically, i.e., virulence can only be achieved through obligate mutualism. Collective pathogenesis has not been previously documented for bacterial pathogens, but an analogous observation has been made in multipartite viruses during infection of beans ${ }^{41}$. This is not to claim that effector arsenals are naturally found distributed among multiple single-effector clones, but it proves the plausibility of collective action contributing to pathogenesis. Collective virulence is also 
consistent with reports of polyclonal and polymicrobial infections, which are best documented in human systems but are becoming increasingly noted in plant systems ${ }^{42-47}$. Specifically for $P$. syringae, collective virulence could help us understand some of its more intriguing life-history traits, such us the transition from environmental to host-associated stages, the maintenance of genetic diversity in the species complex, and host transitions. For example, the co-occurrence of multiple low fitness strains on a new host could collectively support each other until one lineage adapts to the new niche and sweeps through the population. This could be particularly important if one strain carries and ETI-eliciting effector and the other strain carries another effector that suppresses that ETI response $27,30,48-53$

Most studies of cooperation in microbes have been based on centralized sourcing of public goods, wherein one strain incurs the total cost of producing the public good ${ }^{54}$. Our case differs in that no individual strain is paying the cost of producing all the public goods, yet at the same time, no strain is fit without the collective action of multiple strains secreting public goods. This suggests that public goods can act in complementation if provided by multiple members of the community. Our observation of collective virulence is in line with reports of the prevalence of positive pairwise interactions among microbes ${ }^{55,56}$. There are also multiple lines of evidence showing how cooperation is favoured to evolve and be maintained when public goods are encoded by highly mobile genetic elements ${ }^{57}$. These results are in accord with the Black Queen Hypothesis, where it is advantageous to lose essential yet costly functions (e.g., gene products) so long as those functions are produced by other members of the population or community and are public ${ }^{58}$. The loss of the essential public goods will be selectively favored for individual 
strains until an equilibrium community is reached in which loss is balanced by the availability of the public goods.

Metaclone analysis allowed us to clearly demonstrate how a single ETI eliciting effector can negate the fitness benefits of the rest of the effector repertoire, i.e., ETI-elicitation can be thought of as epistatic or dominant. This finding emphasizes the effectiveness of ETI in limiting disease outbreaks, particularly in light of the recent finding that nearly all $P$. syringae strains carry at least one effector that has the potential to elicit ETI ${ }^{26}$. Consequently, pathogens must be under tremendous selective pressure to avoid or suppress these ETI responses, which may explain the very high rate of effector degeneration and loss ${ }^{22}$ and the apparent prevalence of ETI suppressing effectors $^{27,30,48-53}$.

We have also demonstrated that the public goods benefits are largely independent of the genetic background that carries them by showing how collective virulence emerges when effectors are moved into a divergent con-specific strain and even a different species. Our use of the PmaES4326 metaclones reveals that there are not only multiple paths towards virulence on specific hosts, but that the effectors are highly portable across strains, assuming that the receiving strain can exploit these virulence factors (i.e., have a secretion system capable of secreting effectors). These results are consistent with the many cases of apparent independent evolution of specific host associations in P. syringae (i.e., strains causing disease on the same host can often be found in multiple divergent clades) ${ }^{18}$. 
The $P$. fluorescens metaclone demonstrates that effectors (and their associated secretion system) are sufficient to fundamentally change the nature of host-microbe interactions. It is striking that a species adapted to the rhizosphere and commonly used as a plant growth-promoter can be

301

transformed into a highly virulent pathogen by the acquisition of so few genes. This is particularly notable given that the spray inoculation methods used in the study requires the strains to migrate across the leaf surface to the stomatal openings, transit into the leaf apoplast, move into direct contact with the plant cells, inject effectors, and thrive in the apoplastic environment, which presumably is very different than the rhizosphere where $P$. fluorescens is most frequently found. The interaction spectrum separating mutualists from pathogens is clearly quite labile, a finding that further supports the challenges in defining "what is a pathogen" ${ }^{59-61 .}$ While debate on this fundamental question has largely focused on the fact that the outcome of a host-microbe interaction depends on the specific strain, host, and environment, our study demonstrates that even relatively small changes in the genetic composition of the microbiota can result in dramatic changes in the interaction outcome.

The traditional study of pathogenesis since the development of Koch's postulate has focused on the virulence potential of individual strains and largely ignored the potential impact of polyclonal and polymicrobial diversity and interactions. Our observations concretely illustrate that there are more paths to disease than the traditional view of a single virulent strain. In fact, pathogenesis can be a property of a population or community since its collective diversity and interactions can determine the fate of a host-microbe interaction. If injected or secreted virulence factors act as public goods even when delivered from separate strains, we must begin to recognize the 
320 collective output of populations, metapopulations and communities rather than simply focus on

321 single isolates. This view supports our rapidly increasing understanding of the critical role played

322 by the collective microbiota in host health and dysbiosis ${ }^{62}$, and how meta-population dynamics

323 can tip the balance between ETI and host susceptibility ${ }^{63}$. We believe that the metaclone system

324 will open venues for studying the individual components of complex interactions, while allowing

325 for a comprehensive view of effector diversity across the $P$ syringae species complex. Further

326 work is needed to explore the non-independence of community assembly, the distribution of

327 fitness benefits and costs, the mechanisms and level of functional redundancy, the role of clonal

328 competition, diminishing returns epistasis, and other mechanisms driving the acquisition and

329 maintenance of effector diversity among populations and communities. 


\section{References}

3331 Buttner, D. Behind the lines-actions of bacterial type III effector proteins in plant cells. FEMS Microbiol Rev 40, 894-937, doi:10.1093/femsre/fuw026 (2016).

3352 Khan, M., Seto, D., Subramaniam, R. \& Desveaux, D. Oh, the places they'll go! A survey 336 of phytopathogen effectors and their host targets. Plant J 93, 651-663, 337 doi:10.1111/tpj.13780 (2018).

3383 Wei, H. L. \& Collmer, A. Defining essential processes in plant pathogenesis with Pseudomonas syringae pv. tomato DC3000 disarmed polymutants and a subset of key type III effectors. Mol Plant Pathol 19, 1779-1794, doi:10.1111/mpp.12655 (2018).

3414 Wei, C. F. et al. A Pseudomonas syringae pv. tomato DC3000 mutant lacking the type III 342 effector HopQ1-1 is able to cause disease in the model plant Nicotiana benthamiana. 343 Plant J (2007).

3445 Thomas, W. J., Thireault, C. A., Kimbrel, J. A. \& Chang, J. H. Recombineering and 345 stable integration of the Pseudomonas syringae pv. syringae $61 \mathrm{hrp} / \mathrm{hrc}$ cluster into the 346 genome of the soil bacterium Pseudomonas fluorescens Pf0-1. Plant J 60, 919-928, 347 doi:10.1111/j.1365-313X.2009.03998.x (2009).

3486 Evans, A. S. Causation and disease: the Henle-Koch postulates revisited. Yale J Biol Med 349 49, 175-195 (1976).

3507 Hanson, R. P. Koch is dead. J Wildl Dis 24, 193-200, doi:10.7589/0090-3558-24.2.193 $351 \quad$ (1988).

3528 Falkow, S. Molecular Koch's postulates applied to bacterial pathogenicity--a personal 353 recollection 15 years later. Nat Rev Microbiol 2, 67-72, doi:10.1038/nrmicro799 (2004). 
3549 Hosainzadegan, H., Khalilov, R. \& Gholizadeh, P. The necessity to revise Koch's

355 postulates and its application to infectious and non-infectious diseases: a mini-review.

356 Eur J Clin Microbiol Infect Dis 39, 215-218, doi:10.1007/s10096-019-03681-1 (2020).

35710 Vonaesch, P., Anderson, M. \& Sansonetti, P. J. Pathogens, microbiome and the host:

358 emergence of the ecological Koch's postulates. FEMS Microbiol Rev 42, 273-292,

359 doi:10.1093/femsre/fuy003 (2018).

36011 Friesen, M. L. Social evolution and cheating in plant pathogens. Annu Rev Phytopathol

$361 \quad 58,55-75$, doi:10.1146/annurev-phyto-010820-012740 (2020).

36212 Rundell, E. A., McKeithen-Mead, S. A. \& Kazmierczak, B. I. Rampant cheating by

363 pathogens? PLoS Pathog 12, e1005792, doi:10.1371/journal.ppat.1005792 (2016).

36413 Jones, J. D. \& Dangl, J. L. The plant immune system. Nature 444, 323-329,

365 doi:10.1038/nature05286 (2006).

36614 Smith, P. \& Schuster, M. Public goods and cheating in microbes. Curr Biol 29, R442-

367 r447, doi:10.1016/j.cub.2019.03.001 (2019).

36815 West, S. A., Diggle, S. P., Buckling, A., Gardner, A. \& Griffin, A. S. The social lives of 369 microbes. Annual Review of Ecology, Evolution, and Systematics 38, 53-77,

$370 \quad$ doi:10.1146/annurev.ecolsys.38.091206.095740 (2007).

37116 West, S. A., Griffin, A. S., Gardner, A. \& Diggle, S. P. Social evolution theory for 372 microorganisms. Nat Rev Microbiol 4, 597-607, doi:10.1038/nrmicro1461 (2006).

37317 West, S. A. \& Buckling, A. Cooperation, virulence and siderophore production in 374 bacterial parasites. Proc Biol Sci 270, 37-44, doi:10.1098/rspb.2002.2209 (2003). 
18 Dillon, M. M. et al. Recombination of ecologically and evolutionarily significant loci maintains genetic cohesion in the Pseudomonas syringae species complex. Genome Biol 20, 3, doi:10.1186/s13059-018-1606-y (2019).

19 Baltrus, D. A., McCann, H. C. \& Guttman, D. S. Evolution, genomics and epidemiology of Pseudomonas syringae: Challenges in Bacterial Molecular Plant Pathology. Mol Plant Pathol 18, 152-168, doi:10.1111/mpp.12506 (2017).

20 Morris, C. E., Monteil, C. L. \& Berge, O. The life history of Pseudomonas syringae: linking agriculture to earth system processes. Annu Rev Phytopathol 51, 85-104, doi:10.1146/annurev-phyto-082712-102402 (2013).

21 Xin, X. F., Kvitko, B. \& He, S. Y. Pseudomonas syringae: what it takes to be a pathogen. Nat Rev Microbiol 16, 316-328, doi:10.1038/nrmicro.2018.17 (2018).

22 Dillon, M. M. et al. Molecular evolution of Pseudomonas syringae type III secreted effector proteins. Front Plant Sci 10, 418, doi:10.3389/fpls.2019.00418 (2019).

23 Lindgren, P. B., Peet, R. C. \& Panopoulos, N. J. Gene cluster of Pseudomonas syringae pv. phaseolicola controls pathogenicity of bean plants and hypersensitivity of nonhost plants. J Bacteriol 168, 512-522 (1986).

24 Cunnac, S. et al. Genetic disassembly and combinatorial reassembly identify a minimal functional repertoire of type III effectors in Pseudomonas syringae. Proc Natl Acad Sci U S A 108, 2975-2980, doi:10.1073/pnas.1013031108 (2011).

25 Barrett, L. G., Bell, T., Dwyer, G. \& Bergelson, J. Cheating, trade-offs and the evolution of aggressiveness in a natural pathogen population. Ecol Lett 14, 1149-1157, doi:10.1111/j.1461-0248.2011.01687.x (2011). 
39726 Laflamme, B. et al. The pan-genome effector-triggered immunity landscape of a hostpathogen interaction. Science 367, 763-768, doi:10.1126/science.aax4079 (2020).

399

400

401

402

403

404

405

406

407

408

409

410

411

$412 \quad 31$

413

414

415

416

$417 \quad 33$

418

27 Wei, H. L. et al. Pseudomonas syringae pv. tomato DC3000 type III secretion effector polymutants reveal an interplay between HopAD1 and AvrPtoB. Cell Host Microbe 17, 752-762, doi:10.1016/j.chom.2015.05.007 (2015).

28 Chakravarthy, S., Worley, J. N., Montes-Rodriguez, A. \& Collmer, A. Pseudomonas syringae pv. tomato DC3000 polymutants deploying coronatine and two type III effectors produce quantifiable chlorotic spots from individual bacterial colonies in Nicotiana benthamiana leaves. Mol Plant Pathol 19, 935-947, doi:10.1111/mpp.12579 (2018).

29 Kvitko, B. H. \& Collmer, A. Construction of Pseudomonas syringae pv. tomato DC3000 mutant and polymutant strains. Methods Mol Biol 712, 109-128, doi:10.1007/978-161737-998-7_10 (2011).

30 Wei, H. L., Zhang, W. \& Collmer, A. Modular study of the type III effector repertoire in Pseudomonas syringae pv. tomato DC3000 reveals a matrix of effector interplay in pathogenesis. Cell Rep 23, 1630-1638, doi:10.1016/j.celrep.2018.04.037 (2018).

31 Engel, C. Scientific disintegrity as a public bad. Perspect Psychol Sci 10, 361-379, doi:10.1177/1745691615577865 (2015).

32 Martel, A. et al. Metaeffector interactions modulate the type III effector-triggered immunity load of <em>Pseudomonas syringae</em>. bioRxiv, 2021.2011.2018.469137, doi:10.1101/2021.11.18.469137 (2021).

33 Silby, M. W. et al. Genomic and genetic analyses of diversity and plant interactions of Pseudomonas fluorescens. Genome Biol 10, R51, doi:10.1186/gb-2009-10-5-r51 (2009). 
41934 Silby, M. W., Winstanley, C., Godfrey, S. A., Levy, S. B. \& Jackson, R. W.

$420 \quad$ Pseudomonas genomes: diverse and adaptable. FEMS Microbiol Rev 35, 652-680,

421

422

423

424

425

426

427

428

429

430

431

432

433

434

435

436

437

438

439 doi:10.1111/j.1574-6976.2011.00269.x (2011).

35 Haney, C. H., Samuel, B. S., Bush, J. \& Ausubel, F. M. Associations with rhizosphere bacteria can confer an adaptive advantage to plants. Nat Plants $\mathbf{1}$, doi:10.1038/nplants.2015.51 (2015).

36 Brogden, K. A., Guthmiller, J. M. \& Taylor, C. E. Human polymicrobial infections. Lancet 365, 253-255, doi:10.1016/s0140-6736(05)17745-9 (2005).

37 Nelson, A., De Soyza, A., Perry, J. D., Sutcliffe, I. C. \& Cummings, S. P. Polymicrobial challenges to Koch's postulates: ecological lessons from the bacterial vaginosis and cystic fibrosis microbiomes. Innate Immun 18, 774-783, doi:10.1177/1753425912439910 (2012).

38 Tay, W. H., Chong, K. K. \& Kline, K. A. Polymicrobial-host interactions during infection. J Mol Biol 428, 3355-3371, doi:10.1016/j.jmb.2016.05.006 (2016).

39 Isenberg, H. D. Pathogenicity and virulence: another view. Clin Microbiol Rev 1, 40-53, doi:10.1128/cmr.1.1.40 (1988).

40 Schneider, D. J. \& Collmer, A. Studying plant-pathogen interactions in the genomics era: beyond molecular Koch's postulates to systems biology. Annu Rev Phytopathol 48, 457479, doi:10.1146/annurev-phyto-073009-114411 (2010).

41 Sicard, A. et al. A multicellular way of life for a multipartite virus. Elife 8, e43599, doi:10.7554/eLife.43599 (2019). 
44042 Canaday, C., Wyatt, J. \& Mullins, J. Resistance in broccoli to bacterial soft rot caused by

$441 \quad$ Pseudomonas marginalis and fluorescent Pseudomonas species. Plant Dis 75, 715-720

$442 \quad(1991)$.

44343 Moura, M. L., Jacques, M. A., Brito, L. M., Mourão, I. M. \& Duclos, J. Tomato pith $444 \quad$ necrosis (tpn) caused by $P$. corrugata and $P$. mediterranea: severity of damages and crop 445 loss assessment. Acta Hort. 695, 365-372, doi:10.17660/ActaHortic.2005.695.45 (2005).

44644 Kůdela, V., Krejzar, V. \& PáNKoVá, I. Pseudomonas corrugata and Pseudomonas 447 marginalis associated with the collapse of tomato plants in rockwool slab hydroponic $448 \quad$ culture. Plant Prot Sci 46, 1-11 (2011).

44945 Lamichhane, J. R. \& Venturi, V. Synergisms between microbial pathogens in plant $450 \quad$ disease complexes: a growing trend. Front Plant Sci 6, 385, doi:10.3389/fpls.2015.00385 (2015).

45246 Rodrigues, L. M. R., Sera, G. H., Filho, O. G., Beriam, L. O. S. \& de Almeida, I. M. G. First report of mixed infection by Pseudomonas syringae pathovars garcae and tabaci on coffee plantations. Bragantia 74, 543-549, doi:https://doi.org/10.1590/16784499.2016.399. (2017).

47 Karasov, T. L. et al. Arabidopsis thaliana and Pseudomonas pathogens exhibit stable associations over evolutionary timescales. Cell Host Microbe 24, 168-179 e164, doi:10.1016/j.chom.2018.06.011 (2018). acylated target, RIN4, from Arabidopsis membranes to block RPM1 activation. Proc Natl Acad Sci U S A 102, 6496-6501, doi:10.1073/pnas.0500792102 (2005). 
46249 Ritter, C. \& Dangl, J. L. Interference between two specific pathogen recognition events 463 mediated by distinct plant disease resistance genes. Plant Cell 8, 251-257, $464 \quad$ doi:10.1105/tpc.8.2.251 (1996).

46550 Jamir, Y. et al. Identification of Pseudomonas syringae type III effectors that can 466 suppress programmed cell death in plants and yeast. Plant J 37, 554-565 (2004).

46751 Guo, M., Tian, F., Wamboldt, Y. \& Alfano, J. R. The majority of the type III effector 468 469 470 52 Gimenez-Ibanez, S. et al. Differential suppression of Nicotiana benthamiana innate 471 472 473 474 475 47654 Cressler, C. E., Mc, L. D., Rozins, C., J, V. D. H. \& Day, T. The adaptive evolution of 477

53 Wilton, M. et al. The type III effector HopF2Pto targets Arabidopsis RIN4 protein to immune responses by transiently expressed Pseudomonas syringae type III effectors. Front Plant Sci 9, 688, doi:10.3389/fpls.2018.00688 (2018). promote Pseudomonas syringae virulence. Proc Natl Acad Sci U S A 107, 2349-2354, doi:10.1073/pnas.0904739107 (2010).

\section{1} virulence: a review of theoretical predictions and empirical tests. Parasitology 143, 915930, doi:10.1017/s003118201500092x (2016).

55 Oña, L. et al. Obligate cross-feeding expands the metabolic niche of bacteria. Nat Ecol Evol, doi:10.1038/s41559-021-01505-0 (2021).

Kehe, J. et al. Positive interactions are common among culturable bacteria. Sci Adv $\mathbf{7}$, eabi7159, doi:10.1126/sciadv.abi7159 (2021). 
48357 Lee, I. P. A., Eldakar, O. T., Gogarten, J. P. \& Andam, C. P. Bacterial cooperation 484 through horizontal gene transfer. Trends Ecol Evol, doi:10.1016/j.tree.2021.11.006 $485 \quad(2021)$.

48658 Morris, J. J., Lenski, R. E. \& Zinser, E. R. The Black Queen Hypothesis: evolution of 487 dependencies through adaptive gene loss. mBio 3, doi:10.1128/mBio.00036-12 (2012).

48859 Casadevall, A. \& Pirofski, L. A. What is a pathogen? Ann Med 34, 2-4 (2002).

48960 Casadevall, A. \& Pirofski, L. A. The damage-response framework of microbial 490 pathogenesis. Nat Rev Microbiol 1, 17-24, doi:10.1038/nrmicro732 (2003).

49161 Méthot, P. O. \& Alizon, S. What is a pathogen? Toward a process view of host-parasite 492 49362 Gilbert, J. A. et al. Microbiome-wide association studies link dynamic microbial $494 \quad$ consortia to disease. Nature 535, 94-103, doi:10.1038/nature18850 (2016).

49563 Martel, A. et al. The ETS-ETI cycle: evolutionary processes and metapopulation 496 dynamics driving the diversification of pathogen effectors and host immune factors. Curr 497 Opin Plant Biol 62, 102011, doi:10.1016/j.pbi.2021.102011 (2021).

498 499 
a

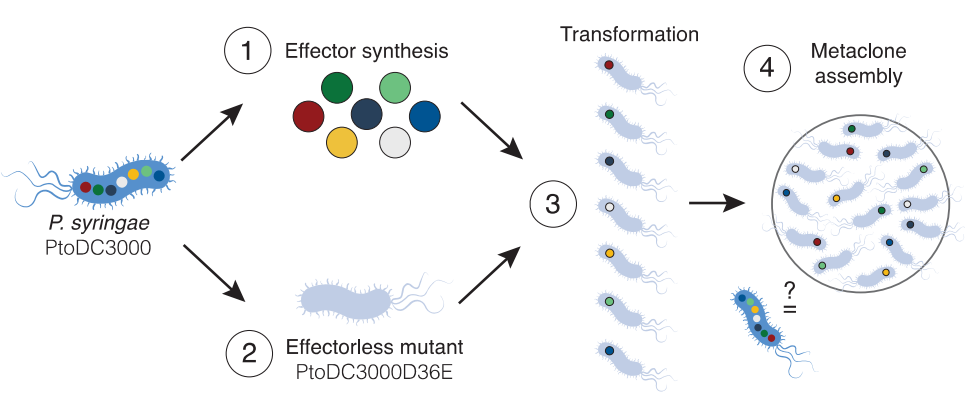

b

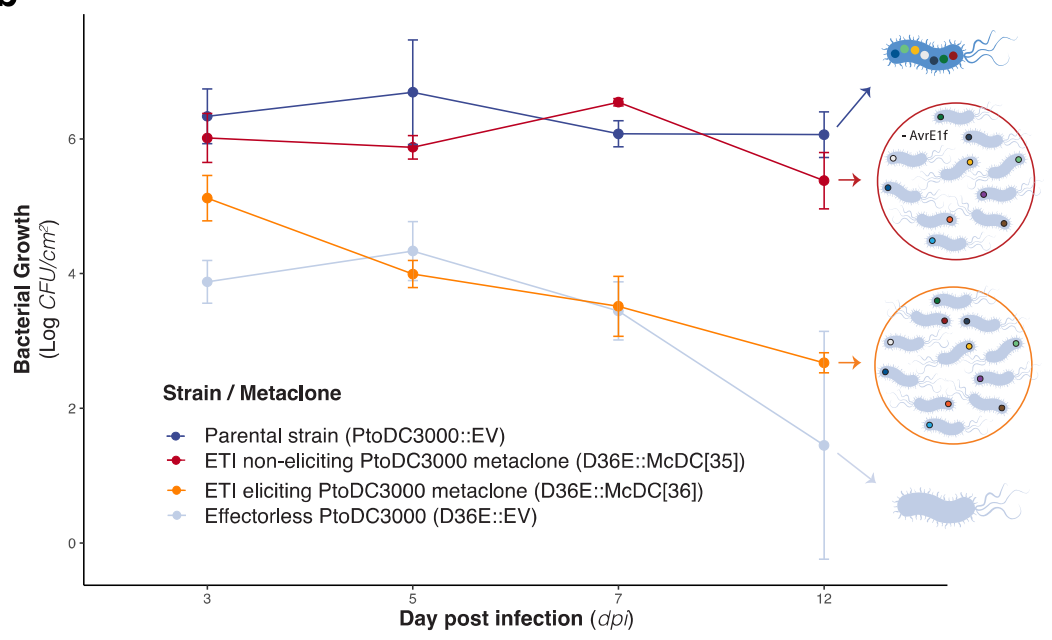

Fig. 1. Metaclones of $\boldsymbol{P}$. syringae allow for the collective emergence of population virulence.

a, Schematic representation of $P$. syringae metaclone construction. (1) Effector gene sequences of a given parental $P$. syringae strain (PtoDC3000) are synthesized and cloned into a broad hostrange vector. (2) The polymutant strain PtoDC3000D36E (here D36E), constructed by Wei et al., 27 is a derivate from PtoDC3000 that lacks all well-expressed effector genes but retains a functional T3SS. (3) Each synthesized effector is individually transformed into D36E. (4) A

508 library of D36E::effector lines is assembled into a metaclone - a population of isogenic strains 509 that differ only by their carriage of a single effector and that collectively mirrors the effector 510 content of its parental strain. b, Virulence of PtoDC3000 is recapitulated collectively by its 511 metaclone on A. thaliana. Endophytic in-planta bacterial growth measured via spray infection 
512 assays at days $3,5,7$, and 12 post-infection is shown for the wildtype pathogen PtoDC3000

513 harboring an empty vector (PtoDC3000::EV), the effector-less D36E harboring an empty vector

514 (D36E::EV), an ETI-eliciting D36E metaclone D36E::McDC[36], and a non-ETI-eliciting

515 metaclone lacking AvrE1f, D36E::McDC[35]. Diagrams on the right represent the corresponding

516 population and genetic makeup of each infection. The non-ETI-eliciting metaclone consistently

517 shows higher fitness than its counterpart metaclone capable of mounting a host immune

518 response. Error bars correspond to standard deviation from the mean of 4 biological replicates

519 per treatment at each timepoint (Supplementary File 2).

520

521 

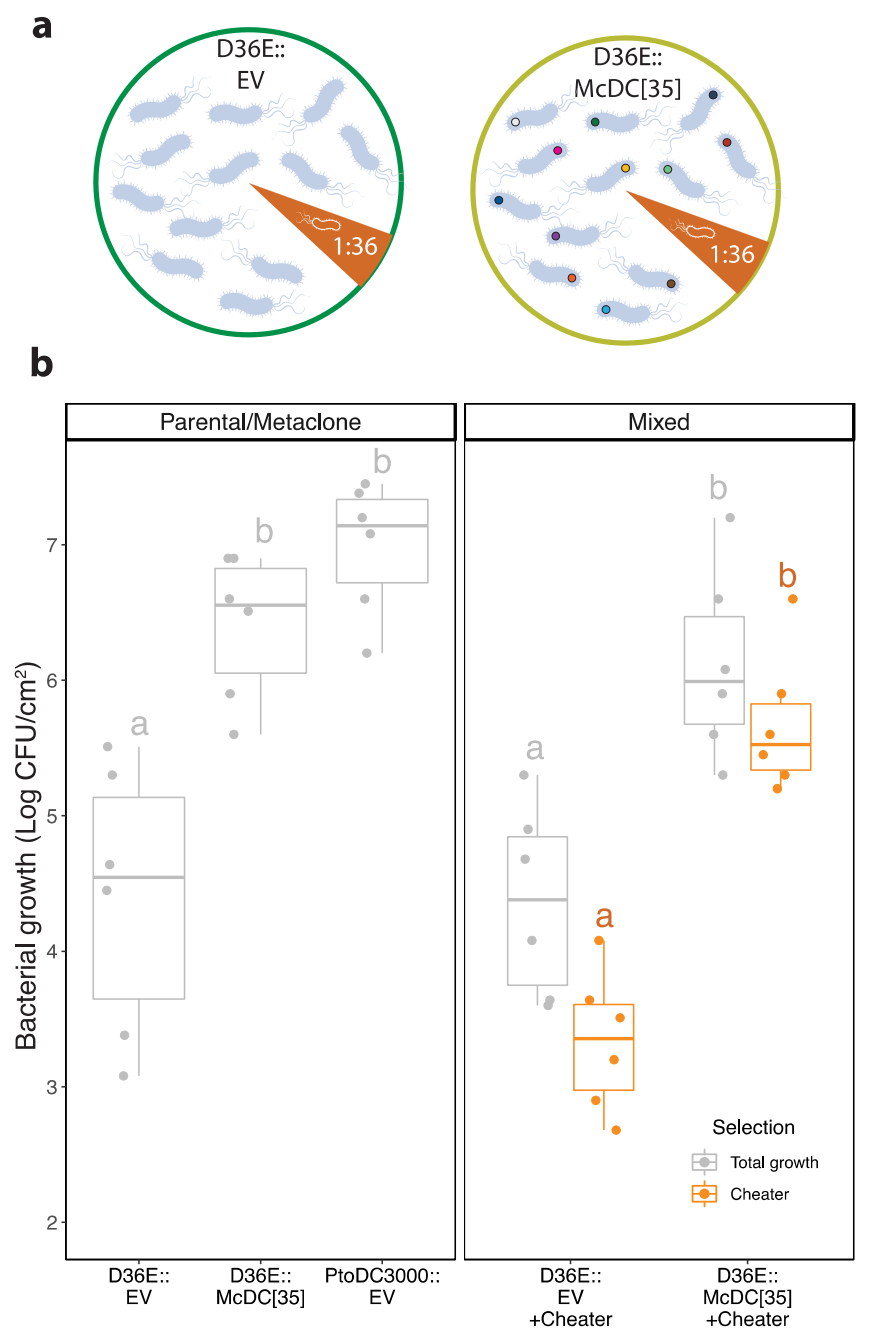

523 Fig. 2. Exploitation of collective virulence by unfit, cheater strains in-planta. a, Composition

524 of two populations mixed with a true, effector-less cheater D36E::pUCP20TC(EV) (shown as the 525 orange wedge) at the same relative abundance (1:36). In the effector-less population (dark 526 green), the cheater strain is mixed only with D36E harboring an empty pBBR1MCS-2 vector 527 (35:36), while in the metaclone population the cheater is complemented with the metaclone D36E::McDC[35] expressing the effector repertoire of the pathogen PtoDC3000 (light green). b,

529 Bacterial endophytic growth at 7 dpi for mixed populations and controls. The emergence of 530 collective virulence in the metaclone D36E::McDC[35] (Mixed panel) drives a significant 
531 increase in fitness of the cheating strain (orange) comparatively to its performance in the absence

532 of effectors in the control population (D36E::EV+cheater). Letters represent statistical

533 significance groups, estimated separately for cheating (orange) and non-cheating portions (grey)

534 via ANOVA and post-hoc Tukey-Kramer honest significant difference (HSD) comparisons

535 performed with a 95\% confidence interval (Supplementary File 2).

536

537

538

539

540

541

542

543

544

545

546 
a

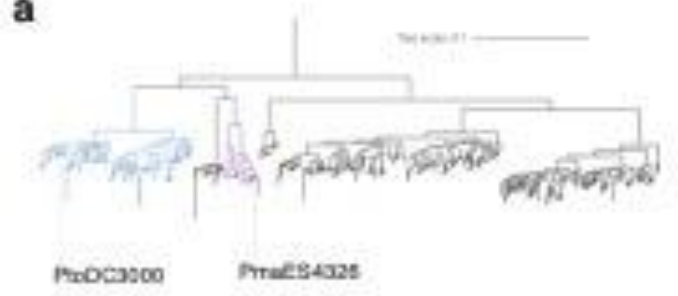

c

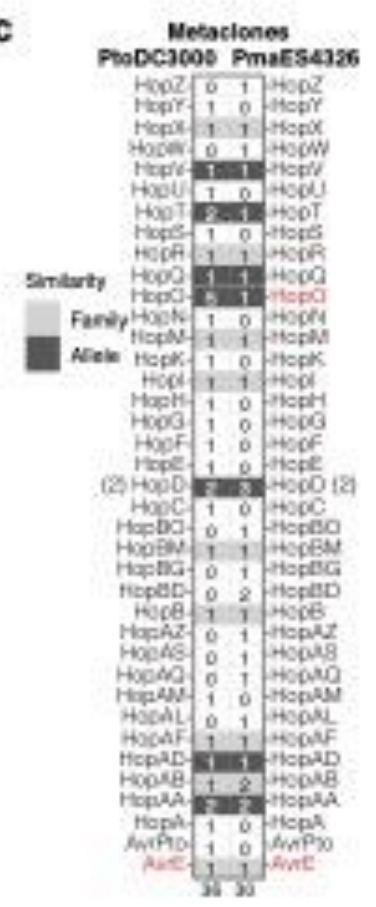

b
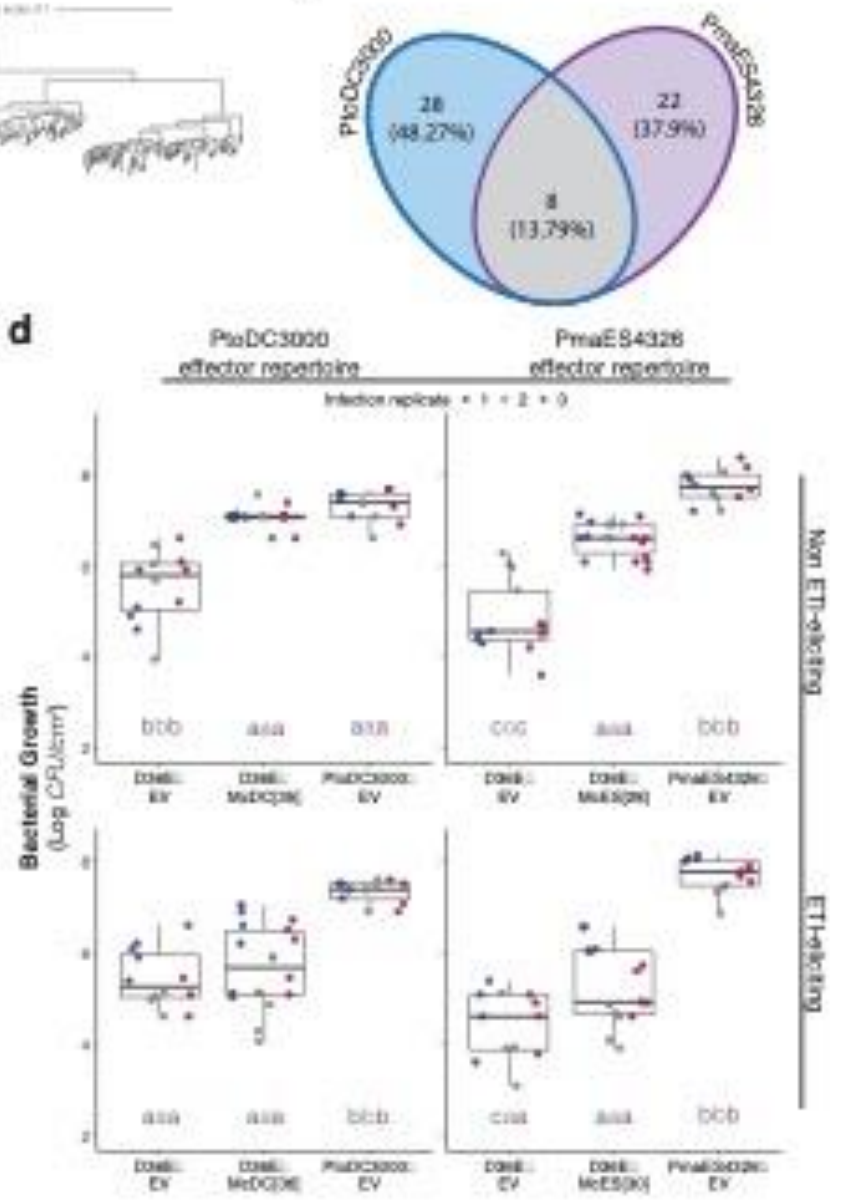

Fig. 3. Portability of pathogenic effector repertoires. a, Core genome, maximum likelihood phylogeny of host-associated strain in the $P$. syringae species complex displays evolutionary relationship between two pathogens of $A$. thaliana, PtoDC3000 and PmaES4326, clustering in divergent clades -phylogroups- 1 (blue) and 5 (purple), respectively. Nodes with distance $<0.01$ are collapsed. Modified from ${ }^{22} \cdot \mathbf{b}$, Venn diagram showing the distribution of PtoDC3000 and

553 PmaES4326 allele-level effector repertoires used in this study. c, Comparison of PtoDC3000 and 554 PmaES4326 effector families. Numbers inside boxes correspond to the total number of unique 555 alleles per family carried by each strain, used for metaclone assembly. Light grey highlighting indicates the two strains share an effector from the same family, while dark grey indicates allelic 
557 identity. Red labeling of an effector indicates a strain-specific ETI-eliciting allele. PtoDC3000

558 and PmaES4326 share two alleles in the HopD family. Additional information in Extended Data

559 Table 1 and Supplementary Table 1. d, Effectors native to the genome of PmaES4326 can

560 drive collective virulence in the distant relative D36E. In-planta bacterial fitness was quantified

5616 dpi for three independent infection replicates. ETI-eliciting and non-ETI-eliciting D36E

562 metaclones of PtoDC3000 and PmaES4326 were compared to the effectorless strain D36E::EV

563 and their corresponding parental strains carrying empty vectors. Similar to the successful

564 PtoDC3000 metaclone, the non-ETI-eliciting PmaES4326 metaclone D36E::McES[28] reaches

565 collective virulence with significantly higher growth than that of the effectorless D36E strain. All

566 comparisons were performed within each infection replicate, and letters represent significant

567 groups estimated via ANOVA and post hoc Tukey-Kramer honest significant difference (HSD)

568 comparisons performed with a confidence level of $95 \%$ for 3 independent experiments

569 (Supplementary File 2).

570

571 


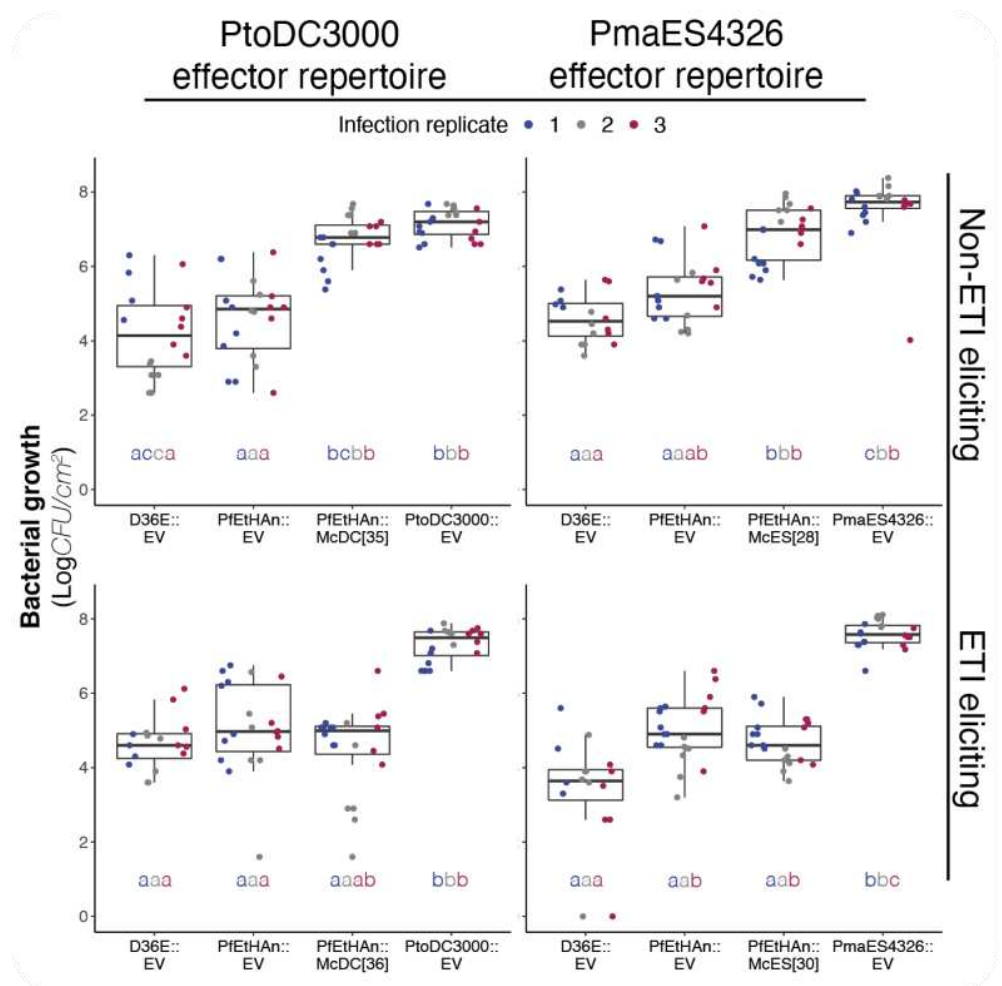

Fig. 4. Effectors are sufficient for converting a beneficial strain into a pathogen. Metaclones

574 constructed by moving the effectors of PtoDC3000 and PmaES4326 individually into the plant 575 growth-promoting strain $P$. fluorescens Pf-0 EtHAn (carrying a $P$. syringae T3SS) achieve high

576 virulence in A. thaliana. Three independent spray infection assays were performed for ETI-

577 eliciting PfEtHAn metaclones PfEtHAn::McDC[36] and PfEtHAn::McES[30], non-ETI-eliciting

578 PfEtHAn metaclones PfEtHAn::McDC[35] and PfEtHAn::McES[28], and control strains

579 harboring an empty vector. Growth in-planta is reported at 6 dpi. All comparisons were

580 performed within each infection replicate, and letters represent significance groups estimated via

581 ANOVA and post hoc Tukey-Kramer honest significant difference (HSD) comparisons 
582 performed with a confidence level of $95 \%$ for 3 independent experiments (Supplementary File $5832)$.

Methods

Effector Selection and Synthesis: Clones were designed as described in ${ }^{26}$. All construct sequences were chemically synthesized by Gene Universal Inc, USA, and synthesized into a Gateway® donor vector harboring a gentamycin resistance marker (pDONR207). While PtoDC3000 has 47 annotated effector alleles ${ }^{22}$, a number of these are known to be pseudogenes or are poorly expressed. Wei et al. ${ }^{27}$ deleted 36 of the 47 effectors from PtoDC 3000 to create the 592 effector polymutant strain D36E, which was used as the genetic background for the metaclones. 593 We elected to generate clones of only the 36 knocked-out PtoDC3000 effectors since the 594 remaining loci are either weak expressors or pseudogenes and still encoded in the D36E genome. 595 The effector HopT2, deleted in D36E was not recognized through our methods and was therefore not included in 596 this study. Three HopO alleles deleted form D36E (HopO1-1, HopO1-2, HopO1-2), correspond 597 to four alleles based on our methods (HopO1a, HopO1b, HopO1c, HopO1d). PmaES4326 has an 598 effector repertoire of 33 effector alleles, of which three were excluded from the metaclone due to 599 lack of expression data (Extended Data Table 1, Supplementary Table 1). Of the nine alleles 600 shared by PtoDC3000 and ES4326 (Fig. 3b), eight are well-expressed hence were reported to be 601 deleted from D36E ${ }^{27}$, and seven do not elicit ETI in A. thaliana (Supplementary Table 1). For 602 this study, we only considered PtoDC3000 effectors that were deleted/disrupted in the 
603

604

605

606

607

608

609

610

611

612

613

614

615

616

617

618

619

620

621

622

623

624

4

construction of D36E (36 alleles) and effectors of PmaES4326 with expression data available (30 alleles) (Extended Data Table 1, Fig. 3b-c).

Effector transformation and tri-parental mating: Each pDONR207 vector was transformed into chemically competent Escherichia coli DH5 $\alpha$ cells. To confirm insert sizes, plasmids were purified with Thermo Scientific GeneJet Plasmid Miniprep Kit, NotI sites flanking the construct were targeted for restriction enzyme digestion, and band sizes were confirmed by agarose electrophoresis. Gateway® cloning (LR reaction) was performed in order to transfer the construct insert from the donor vector into a low copy broad-host-range expression vector (pBBR1MCS-2) with a kanamycin resistance marker, and insert sizes were again confirmed via NotI and EcoRV (from pBBR1MCS-2 backbone) digestions.

Tri-parental mating was used to deliver each of the constructs in pBBR1MCS-2 into either the $P$. syringae polymutant strain D36E or the $P$. fluorescens Pf01EtHAn strain, with their native rifampicin or tetracycline resistance respectively. For this, the strain E. coli HB101 (RK600 chloramphenicol resistant) was used as a helper and double antibiotic selection including kanamycin was used to isolate successful colonies after plasmid delivery. Control strains (PtoDC3000::EV, PmaES4326::EV, PfEtHAn::EV and D36E::EV) were built by delivering an empty pBBR1MCS-2 vector into the "wildtype" strain and consistently maintaining both antibiotics for proper selection. A pUCP20TC non-gateway empty vector was used to construct the true cheater strain D36E::pUCP20TC(EV) 

640 hours).

Bacterial growth conditions: All Pseudomonas spp. strains were grown in selective King's B agar (KB). Media was supplemented with antibiotics as follows: $50 \mathrm{ug} / \mathrm{mL}$ of rifampicin for any PtoDC3000 or D36E strain/metaclone, $8 \mathrm{ul} / \mathrm{mL}$ of tetracycline for any strain /metaclone of Pf01EtHAn, and $100 \mathrm{ug} / \mathrm{mL}$ of streptomycin for PmaES4326. To maintain the plasmid in transformed strains or populations harboring either a pBBR1MCS-2 or pUCP20TC construct, they were grown in media additionally supplemented with $50 \mathrm{ug} / \mathrm{mL}$ kanamycin or $8 \mathrm{ul} / \mathrm{mL}$ of tetracycline, respectively.

In-vitro bacterial growth assays: Growth curves were performed for D36E::effector lines before assembling the metaclones D36E::McDC[35] and D36E::McDC[36] (Fig. S1). Each strain was grown in liquid $\mathrm{KB}$ media with kanamycin overnight. Optical densities (OD) were measured and adjusted to 0.05 and a starting volume of $150 \mathrm{ul}$, which were transferred in triplicate to 96-well plates, avoiding edge wells. Growth assays were performed in a microplate reader (ICG12 robot manufactured by S\&P Robotics) set to shake and quantify OD at $600 \mathrm{~nm}$ every 2 hours while maintaining a constant temperature of $30^{\circ} \mathrm{C}$ for 60 hours $(\sim 10$ generations/24

Plant growth conditions: Arabidopsis thaliana ecotype Col-0 plants were grown under controlled conditions until ready for infection assays. Seeds are sowed into Sunshine ${ }^{\circledR}$ Mix \#1, LC1 (75-85\% sphagnum peat moss, perlite, dolomite, limestone, $\mathrm{pH}$ adjusted) and kept domed until germination. On week 2, seedlings were thinned and spaced to avoid overlap at full size. Regular watering and growth chamber conditions (12-hour dark photoperiod at $21^{\circ} \mathrm{C}$ and $12-$ 
647 hour at $20^{\circ} \mathrm{C}$ ) were maintained until plants reach 3-4 weeks when they are transferred into the 648 laboratory for infection assays.

649

650

651

652

653

654

656

657

658

659

660

661

662

663

664

665

666

667

668

669

Metaclone assembly: Individual strains were streaked from $-80^{\circ} \mathrm{C}$ glycerol stocks and grown overnight in the corresponding antibiotics at $28^{\circ} \mathrm{C}$. Liquid cultures were inoculated and grown shaking overnight until saturation, when OD at $600 \mathrm{~nm}$ was measured. If necessary, optical densities were adjusted by spinning down the cultures for 5 minutes at $4600 \mathrm{rpm}$ and correcting their final volume to reach equal concentrations. For any given metaclone, equal volumes of individual strains were mixed to produce a single liquid culture with all corresponding antibiotics, which is then vortexed at low speed until well-mixed, and finally stocked or plated. Note that for instances where more than one allele of the same cluster (e.g., HopR1g) was annotated and included in this study, the final concentration of its corresponding D36E or EtHAn metaclone was adjusted accordingly.

Spray infection assays: All strains and metaclones used for spray inoculation assays were taken through two growth cycles to help synchronize growth and guarantee infections were made with fresh culture. A first streak from $-80^{\circ} \mathrm{C}$ glycerol stocks in the corresponding antibiotics was followed by 30-48 hours of growth at room temperature. Following, bacteria was resuspended in 10mM MgSO4 and streaked onto fresh plates to form lawns. For metaclones, either fresh wellmixed liquid culture was plated or full cryovial stocks were thawed and immediately plated. Heavy metaclone lawns were recovered by adding 4mL of $1 \mathrm{X} \mathrm{KB}$ media, shaking the plates at $1700 \mathrm{rpm}$, and a final solution of 1:100 was prepared, plated, and grown at $28{ }^{\circ} \mathrm{C}$ for infection. When ready for spray infection, strains and metaclones were recovered from plates, resuspended 
670

671

672

673

674

675

676

677

678

679

680

681

682

683

684

685

686

687

688

689

690

691

692

in a solution of $10 \mathrm{mM} \mathrm{MgSO}_{4}$ and $0.04 \%$ Silwet L-77, and were sprayed at an $\mathrm{OD}_{600}=2$. A volume of approximate $2.5 \mathrm{~mL}$ of bacterial solution was sprayed into each plant using Preval sprayers. Immediately after spraying, plants were domed to maintain consistent humidity conditions until the completion of each experiment. The progression of diseases was monitored by bacterial growth assays. Day 0 measurements of $\mathrm{OD}_{600}$ were taken only with the purpose of adjusting to the desired value but are not included in figures as they represent the sprayed solution but not in-planta growth.

Leaf syringe infiltration assays: Similar to spray infections, syringe pressure infiltrations were performed with strains or populations on active log phase, grown overnight in solid KB media supplemented with their corresponding antibiotics. On the day of infection, plants were marked and domed at least one hour prior to infection to incentivize stomatal opening. All bacterial treatments were resuspended in $10 \mathrm{mM} \mathrm{MgSO}_{4}$ and diluted to reach a final $\mathrm{OD}_{600}=0.00002$. Using 1cc needle-less sterile syringes, leaves were pressure infiltrated and excess moisture was cleaned. After infection, plants were domed for 1 hour, after which Day 0 measurements were taken, and plants were finally returned to their growth chamber conditions, undomed, until next harvesting timepoint.

In-planta growth assays: Following spray or pressure infiltration infections, in-planta growth assays were carried out to quantify endophytic bacterial fitness. For each infected plant, tissue was harvested from a total of 4 leaves, which were cleaned from excess moisture before coring. An area of $1 \mathrm{~cm}^{2}$ was collected from each leaf using a \#3 corer, 4 cores ( 4 leaves $=1$ plant $)$ were polled together to be sterilized with $70 \%$ ethanol for 10 seconds to dispose of epiphytes in 
control experiments. All 4 harvested cores per plant (typically 5 plants per treatment) were then transferred together into $200 \mathrm{ul}$ of $10 \mathrm{mM} \mathrm{MgSO}_{4}$ with a sterile glass bead to be then grinded in a bead-beater for $2 \mathrm{~min}$, in order to allow for the release and recovery of endophytic bacterial cells residing in the apoplast. The plant-microbe solution was then transferred to the top row of a 96 well-plate, 10-fold serial dilutions were performed by transferring $20 \mathrm{ul}$ of $10 \mathrm{mM} \mathrm{MgSO}_{4}$ into wells with $180 \mathrm{ul}$ of the same $\mathrm{MgSO}_{4}$ solution, and $5 \mathrm{ul}$ of solution across dilutions were plated on solid KB media with the native antibiotic of the corresponding strain or population. Colony counts were performed after $24-48 \mathrm{hrs}$ of growth at $30^{\circ} \mathrm{C}$ and bacterial fitness results were reported as $\log \left(\mathrm{CFU} / \mathrm{cm}^{2}\right)$.

Effector barcode amplification, sequencing, and quantification: Effector specific barcodes were obtained directly from infected leaves via PCR. Primers were designed based on the final pBBRMCS-2 broad-host-range vector containing the synthesized constructs. All barcodes are 8bp long and are at least one mutational step away from each other ${ }^{26}$. Primers were designed to include Nextera overhang adapters to allow for manageable high throughput library preparation (Extended Data Table 2) and produce an amplicon of approximate $\sim 300 \mathrm{bp}$ which only differs across all constructs in their barcode sequence. A modified Dilution and Storage protocol using Thermo Scientific ${ }^{\mathrm{TM}}$ Phire Plant Direct PCR was optimized to allow for storage of the plantmicrobe solution obtained during in-planta bacterial growth assays for subsequent amplification. This strategy was optimized so that amplicon concentrations reflected bacterial densities used for infection to recovery threshold at $\mathrm{OD}_{600}=0.002$ and effector composition of metaclones in sequencing data followed expectations based on the initial molarity of individual effectors in the inoculum. Tissues were stored at $-20^{\circ} \mathrm{C}$. A 2-step, short-cycle PCR reaction was optimized to 
avoid PCR bias, as follows: initial denaturation for 5 minutes at $98^{\circ} \mathrm{C}, 24$ cycles of $[5$ seconds at $98^{\circ} \mathrm{C}$ followed by extension for 5 seconds at $72^{\circ} \mathrm{C}$ ], and 1 minute at $72^{\circ} \mathrm{C}$. Amplicons were column-cleaned using Macherey-Negel ${ }^{\mathrm{TM}}$ NucleoSpin ${ }^{\mathrm{TM}}$ Gel and PCR Clean-up and sent for NextSeq Illumina 150 pair-end sequencing at the Center for the Analysis of Genome Evolution and Function (CAGEF). Trimmomatic ${ }^{64}$ was used to remove sequencing adapters, filter with minimal Phred score of 33 and minimal length of $100 \mathrm{bp}$. Finally, in-house custom python scripts using Biopython ${ }^{65}$ modules were used for quantification of reads matching the effectorspecific barcode sequence strings using regular expressions. Mean Shannon index was estimated to represent diversity in barcode sequence data using the R package vegan ${ }^{66}$.

Public good experiment: Mixed populations were assembled by inoculating KB broth liquid cultures supplemented with rifampicin only and incubating overnight at $30^{\circ} \mathrm{C}$, shaking until saturation. Cultures were spun down and OD600 was normalized across all clones before mixing in equal volumes. Metaclone assembly conditions were followed downstream, keeping only rifampicin selection before infection. For the mock metaclone, D36E harboring an empty pBBR1MCS-2 construct was mixed with D36E harboring a pUCP20TC empty vector at a 35:36 ratio. Spray infections and growth assays were performed as usual with replicate plating of the growth assay 10-fold serial dilution in rifampicin alone, rifampicin plus kanamycin, and rifampicin plus tetracycline to select for total growth, pBBR1MCS-2 construct-harboring growth and pUCP20TC-harboring growth (cheater).

\section{Statistics: To evaluate differences in growth between strains/metaclones, ANOVA were} performed with post-hoc Tukey or Tukey-Kramer HSD tests using a 95\% confidence intervals 
739 All data processing was performed in RStudio v.1.3.1073, using packages in Tidyverse, ggpubr,

740 and multicompView for significant grouping. Source data and statistical analyses for all main

741 figures are reported in Supplementary File 2.

742

743 Code availability

744 Any script used in the analysis of the data presented in this study is available upon request.

745

74664 Bolger, A. M., Lohse, M. \& Usadel, B. Trimmomatic: a flexible trimmer for Illumina

747 sequence data. Bioinformatics 30, 2114-2120, doi:10.1093/bioinformatics/btu170 (2014).

74865 Cock, P. J. et al. Biopython: freely available Python tools for computational molecular

749 biology and bioinformatics. Bioinformatics 25, 1422-1423,

750 doi:10.1093/bioinformatics/btp163 (2009).

75166 Oksanen, J. et al. vegan: Community Ecology Package, < $\underline{\text { http://cran.r- }}$

752 project.org/web/packages/vegan/index.html> (2011).

754 Acknowledgements

755 We thank the members of the Desveaux and Guttman laboratories for their advice and feedback

756 throughout this project, with particular thanks to Alexandre Martel for his assistance with strain

757 transformation and to Marcus Dillon for his insights in effector extraction and construct

758 synthesis. We would also like to thank and acknowledge Alan Collmer and Jeff Chang for

759 providing us with the strains PtoDC3000D36E (CUCPB6119) and Pf01EtHAn, that were 
760 invaluable to this study. This project is supported by Natural Sciences and Engineering Research

761 Council of Canada (NSERC) Discovery Grants (DSG and DD).

\section{Author Contributions}

764 T.R-B., D.D., and D.S.G. designed the project; T.R-B performed transformations, tri-parental 765 matings, metaclones construction, spray infections, in-planta and in-vitro growth assays, primer 766 design, barcode quantification, and analyzed the data; P.W.W. generated sequence data and 767 assisted with many aspects of the project; T.R-B., D.D., and D.S.G. wrote the paper; all authors 768 reviewed and agreed on the manuscript.

770 Competing Interests: The authors declare no competing interests.

\section{Additional Information}

773 Supplementary Information is available for this paper

774 Correspondence and requests for materials should be addressed to Darrell Desveaux

775 (darrell.desveaux@utoronto.ca and) and David Guttman (david.guttman@utoronto.ca).

776 Reprints and permissions information is available at www.nature.com/reprints

$777 \quad$ Extended Data

778 Extended Data Figure 1-5

779 Extended Data Table 1-2 
780 Supplementary File 1. All construct sequences used in this study and associated metadata (Excel 781 format)

782 Supplementary File 2. Source with corresponding statistical analysis outputs (Excel format) 


\section{Supplementary Files}

This is a list of supplementary files associated with this preprint. Click to download.

- Metaclonemsv21ExtDataSuppl.pdf

- SupplementaryFile1.xIsx

- SupplementaryFile2.xlsx 